\title{
Orbit of the young very low-mass spectroscopic binary CHXR $74^{\star}$
}

\author{
V. Joergens ${ }^{1,2}$, M. Janson ${ }^{3}$, and A. Müller ${ }^{2}$ \\ 1 Zentrum für Astronomie Heidelberg, Institut für Theoretische Astrophysik, Albert-Ueberle-Str. 2, 69120 Heidelberg, Germany \\ 2 Max-Planck Institut für Astronomie, Königstuhl 17, 69117 Heidelberg, Germany \\ e-mail: viki@mpia.de \\ 3 Princeton University, Dept. of Astrophysics, 4 Ivy Lane, Princeton, NJ 08544, USA
}

Received 5 October 2011 / Accepted 24 October 2011

\begin{abstract}
The pre-main sequence star CHXR 74 (M4.25) in Chamaeleon I was found a few years ago to be a very low-mass spectroscopic binary. A determination of its mass would provide a valuable dynamical mass measurement at young ages in the poorly constrained mass regime of $<0.3 M_{\odot}$. We carried out follow-up radial velocity monitoring with UVES/VLT between 2008 and 2011 and high-resolution adaptive-optic-assisted imaging with NACO/VLT in 2008 with the aim of constraining the binary orbit. We present an orbital solution of the system based on the combined radial velocity data set, which spans more than eleven years of UVES monitoring for CHXR 74. The best-fit Kepler model has an orbital period of 13.1 years, zero eccentricity, and a radial velocity semi-amplitude of $2.2 \mathrm{~km} \mathrm{~s}^{-1}$. A companion mass $M_{2} \sin i$ (which is a lower limit due to the unknown orbital inclination $i$ ) of $0.08 M_{\odot}$ is derived by using a modeldependent mass estimate for the primary of $0.24 M_{\odot}$. The binary separation $\left(a_{1} \sin i+a_{2}\right)$ for an inclination of $90^{\circ}$ is $3.8 \mathrm{AU}$, which corresponds to 23 mas. Complementary NACO/VLT images of CHXR 74 were taken with the aim to directly resolve the binary. While there are marginal signs of an extended point spread function (PSF), we have detected no convincing companion to CHXR 74 in the NACO images. From the non-detection of the companion together with a prediction of the binary separation at the time of the NACO observations, we derive an upper limit for the $K$-band brightness ratio of the two binary components of 0.5 . This allows us to estimate an upper limit of the companion mass of $0.14 M_{\odot}$ by applying evolutionary models. Thus, we confirm that CHXR 74 is a very low-mass spectroscopic binary and constrain the secondary mass to lie within the range of about 0.08 and $0.14 M_{\odot}$. We predict an astrometric signal of the primary between 0.2 and 0.4 mas when taking into account the luminosity of the companion. The Gaia astrometric mission might well be able to solve the astrometric orbit of the primary and in combination with the presented radial velocity data determine an absolute companion mass.
\end{abstract}

Key words. stars: pre-main sequence - binaries: spectroscopic - stars: low-mass - techniques: radial velocities stars: individual: CHXR74

\section{Introduction}

Binaries whose orbital periods are sufficiently short for us to follow their orbital motion in a reasonable time are key astronomical objects because they allow us dynamical mass measurements. Masses are the very foundation of our understanding of star-, brown dwarf- (BD) and planet formation and evolution, e.g. they define the IMF and are the most important input parameter for evolutionary models. A means to search for companions with orbital distances of a few AU are spectroscopic surveys that monitor the radial velocity (RV) shift caused by a close companion. From the spectroscopic orbit alone, only lower mass limits $M \sin i$ can be determined because of the unknown inclination $i$. Spectroscopic binaries for which the astrometric or visual orbit can be determined or that happen to be eclipsing systems allow absolute mass determinations. In the mass regime $<0.3 M_{\odot}$, a few spectroscopic binaries have been detected and their orbits determined (e.g., Basri \& Martín 1999; Stassun et al. 2006; Joergens \& Müller 2007; Joergens et al. 2010; Blake et al. 2008). Furthermore, follow-up RV measurements permitted additional

$\star$ Based on observations obtained at the Very Large Telescope of the European Southern Observatory at Paranal, Chile with UVES in program 65.I-0011(A), 72.C-0653(A), 75.C-0851(C), 77.C-0831(A+D), 380.C-0596(A), 082.C-0023(A), 087.C-0962(B), and with NACO in program 380.C-0596(B). constraints of the orbits of a few close visual BD and very lowmass (VLM) binaries (e.g., Zapatero Osorio et al. 2004; Simon et al. 2006; Konopacky et al. 2010). For masses $<0.3 M_{\odot}$, evolutionary models rely only on very few mass measurements for very young binaries (Mathieu et al. 2007; Stassun et al. 2006). While there used to be little or no overlap between spectroscopic, and, consequently close binaries and visual binaries, particularly at the distance of star-forming regions, the situation is changing owing to recent advances in spatial resolution achieved by adaptive optics $(\mathrm{AO})$ and interferometric instruments.

Within the course of an RV survey for binaries among very young BDs and low-mass stars with UVES/VLT in the Chamaeleon I (Cha I) star-forming cloud, the very low-mass star CHXR 74 (M 4.25, $\sim 0.24 M_{\odot}$ ) was detected to be a spectroscopic binary with a presumably relatively long orbital period (Joergens 2006, 2008).

We have carried out follow-up UVES observations between 2008 and 2011 to constrain the spectroscopic orbit. Furthermore, we have obtained high-resolution AO images with NACO/VLT of CHXR 74 with the aim to directly resolve the binary. We present here the results of these observations, which include the determination of an RV orbit solution and of an upper limit for the brightness ratio. Finally, we derive constraints for the masses of the two binary components and investigate prospects for future dynamical mass measurements. 


\section{CHXR 74}

CHXR 74 was identified as low-mass member of the Cha I star-forming cloud with spectral type M 4.5 by Comerón et al. (1999). CHXR 74 has a brightness of 17.3 mag in $V$ band and 10.2 mag in $K_{\mathrm{S}}$ band (Comerón et al. 1999; Luhman 2004) and is classified as class III object based on its spectral energy distribution, i.e. it has no detected disk (Luhman et al. 2008). The most recent determination of stellar parameters for CHXR 74 yields a spectral type of M4.25, an effective temperature of $T_{\text {eff }}=3234 \mathrm{~K}$, and a bolometric luminosity of $L=0.15 L_{\odot}$ (Luhman 2007). Using these stellar parameters of the unresolved system, we estimate a mass of about $0.25 M_{\odot}$ and an age of about 2 Myr for CHXR 74 based on evolutionary model tracks (Baraffe et al. 1998). We show below that the companion contributes significantly to the total luminosity of the system and that the model-dependent mass of the primary is $0.23-0.24 M_{\odot}$, instead (cf. Sect. 5).

\section{Radial velocities and orbital solution}

Spectroscopic observations of CHXR 74 were carried out between 2000 and 2011 with the Ultraviolet and Visual Echelle Spectrograph (UVES, Dekker et al. 2000) attached to the VLT $8.2 \mathrm{~m}$ KUEYEN telescope at a spectral resolution $\lambda / \Delta \lambda$ of 40000 in the red optical wavelength regime. The RVs were measured from these spectra based on a cross-correlation technique employing telluric lines for the wavelength calibration. Details of the data analysis can be found in Joergens $(2006,2008)$.

The RV measurements for spectra taken between 2000 and 2006 provided evidence that CHXR 74 has a long-period spectroscopic companion (Joergens 2006, 2008). Here, we present new RV data based on UVES spectra taken between 2008 and 2011. Table 1 lists the new RV measurements of CHXR 74 and previous RV data. The latter were reprocessed for this work by using a slightly refined wavelength range for the crosscorrelation to exclude a region that had been found to be contaminated by telluric lines and imperfectly corrected CCD cosmetic blemishes and that introduced additional scatter. The given errors $\sigma_{\mathrm{RV}}$ are the sample standard deviation of two consecutive individual measurements. The RV noise caused by activity is estimated to about $450 \mathrm{~m} \mathrm{~s}^{-1}$ based on the night-to-night rms scatter in the data. This error value is used for finding the $\mathrm{RV}$ orbit solution.

The combined data set spans more than 11 years of RV monitoring for CHXR 74 between 2000 and 2011 (Fig. 2). Starting in 2000, we witnessed first an increase of the RV until 2006 by about $4 \mathrm{~km} \mathrm{~s}^{-1}$ followed by a period of only small RV changes and finally a decrease of the RV in 2011 back to the original RV level.

To identify periodicities in the RV data, we computed the generalized Lomb-Scargle (GLS) periodogram (Zechmeister \& Kürster 2009) and its window function (Fig. 1). For a better visibility of longer periods, the GLS periodogram is plotted with a logarithmic scale for the period.

The GLS periodogram reveals a significant peak at a period of $4785 \mathrm{~d}$ with a false-alarm probability (FAP) of $2.3 \times 10^{-8}$. In addition, there are several peaks at shorter periods but they have FAPs higher than $10^{-3}$ and are not considered to be significant. These peaks are expected to be aliases because the periodogram is a convolution of a present signal in the data and the sampling function of these data (window function). For example, the alias peak in the periodogram at $340 \mathrm{~d}$ is caused by the $365 \mathrm{~d}$ sampling period (yearly alias, highest peak in the window function).
Table 1. RV measurements of CHXR74.

\begin{tabular}{lccc}
\hline \hline Date & HJD & $\begin{array}{c}\mathrm{RV} \\
{\left[\mathrm{km} \mathrm{s}^{-1}\right]}\end{array}$ & $\begin{array}{c}\sigma_{\mathrm{RV}} \\
{\left[\mathrm{km} \mathrm{s}^{-1}\right]}\end{array}$ \\
\hline 20000313 & 2451616.787145 & $15.829^{a}$ & 0.012 \\
20000331 & 2451634.520915 & $15.211^{a}$ & 0.216 \\
20000422 & 2451656.512470 & $15.770^{a}$ & 0.095 \\
20000521 & 2451686.482610 & $14.822^{a}$ & 0.031 \\
20040303 & 2453067.829560 & $17.863^{a}$ & 0.033 \\
20040312 & 2453076.664875 & $18.223^{a}$ & 0.005 \\
20040321 & 2453085.770220 & $18.282^{a}$ & 0.109 \\
20040324 & 2453088.798215 & $18.020^{a}$ & 0.083 \\
20040331 & 2453095.780420 & $19.003^{a}$ & 0.448 \\
20040401 & 2453096.776000 & $18.779^{a}$ & 0.008 \\
20050321 & 2453450.589225 & $19.468^{a}$ & 0.172 \\
20060410 & 2453835.634560 & 18.845 & \\
20060615 & 2453901.522670 & 19.642 & \\
\hline 20080219 & 2454515.798960 & 18.712 & \\
20080307 & 2454532.786240 & 19.202 & \\
20080319 & 2454544.569660 & 19.719 & \\
20090112 & 2454843.867690 & 18.700 & \\
20090114 & 2454845.857240 & 18.595 & \\
20090116 & 2454847.754840 & 18.628 & \\
20110406 & 2455657.749380 & 16.396 & \\
20110527 & 2455708.561260 & 16.168 & \\
20110703 & 2455746.490790 & 15.414 & \\
\hline
\end{tabular}

Notes. Listed are new RV data (bottom panel) and previous RV measurements (top panel), which were re-processed for this work. HJD is given at the middle of the exposure; $\sigma_{\mathrm{RV}}$ is the sample standard deviation of two consecutive measurements. The error of the relative RVs taking into account RV noise caused by activity is estimated to about $450 \mathrm{~m} \mathrm{~s}^{-1}$. An additional error of about $400 \mathrm{~m} \mathrm{~s}^{-1}$ has to be taken into account for the absolute RVs. ${ }^{(a)} \mathrm{RV}$ value is the average of two single consecutive measurements.

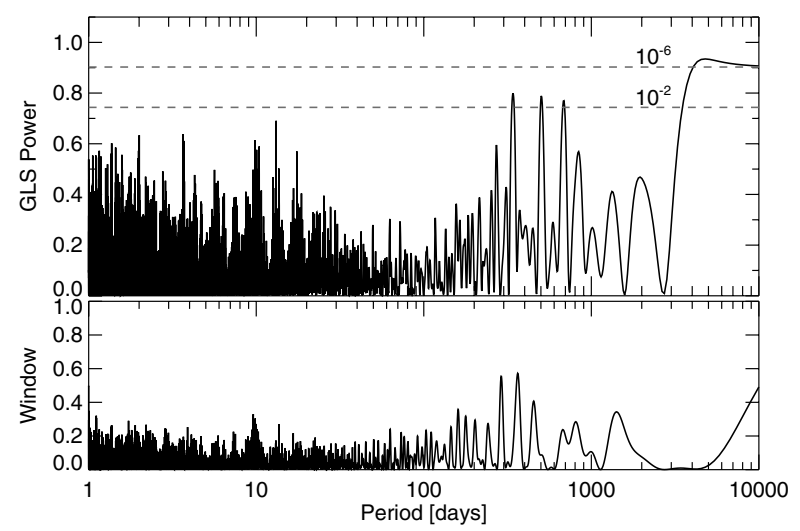

Fig. 1. GLS periodogram (upper plot) of the RV data, and the corresponding window function (lower plot). The two horizontal dashed lines indicate FAP levels of $10^{-2}$ and $10^{-6}$. The highest peak at a period of 4785 days has an FAP of $2.3 \times 10^{-8}$.

We calculated an orbital solution based on the RV data using $\chi^{2}$ minimization. We fitted the four free parameters period $P$, periastron time $T, \mathrm{RV}$ semi-amplitude $K$, and system velocity $V_{0}$. The eccentricity $e$ was fixed to zero in this fit because treating it as free parameter leads to very high eccentricity values, poorly constrained longitudes of periastron, and unreasonable values for the lower limit of the companion mass $M_{2} \sin i$ (several times higher than $M_{1}$ ). We note that such unrealistic results occur for $e>0.2$, while derived orbit parameters for $e \leq 0.2$ are consistent within the errors with the values derived for $e=0$. 


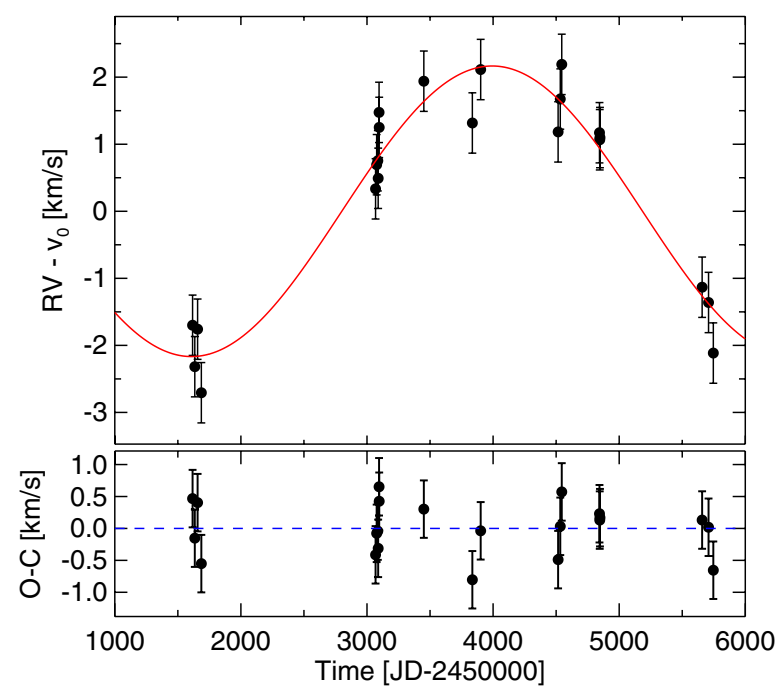

Fig. 2. Upper panel: RV measurements of CHXR 74 between 2000 and 2011 based on UVES/VLT spectra. The system velocity $V_{0}$ was subtracted. The red solid line shows the best-fit Keplerian orbit for a period of 4770 days (13.1 years) and a semi-amplitude of $2.2 \mathrm{~km} \mathrm{~s}^{-1}$. Lower panel: RV residuals.

The best-fit Kepler orbit is shown in Fig. 2. It has a period of 4770 days (13.1 yr) and an RV semi-amplitude of $2.2 \mathrm{~km} \mathrm{~s}^{-1}$. The derived lower limit to the semi-major axis of the primary $a_{1} \sin i$ is $0.95 \mathrm{AU}$. The reduced $\chi^{2}$ of the orbital fit is 0.94 . The complete list of determined orbital elements is given in Table 2.

The minimum mass $M_{2} \sin i$ and the semi-major axis $a_{2}$ of the secondary for a single-lined RV orbit depend on the primary mass. The mass estimate for CHXR 74 based on the luminosity and effective temperature of the unresolved source by applying evolutionary models is $0.25 M_{\odot}$ (cf. Sect. 2). We show in Sect. 5 that the companion contributes significantly to the total luminosity of the system, which leads to a reduced primary mass. Using $M_{1}=0.24 M_{\odot}$, the mass $M_{2} \sin i$ of the companion is determined to be $84 M_{\text {Jup }}\left(0.08 M_{\odot}\right)$ and its semi-major axis $a_{2}$ to be $2.84 \mathrm{AU}$. The errors in $M_{2} \sin i$ and $a_{2}$ given in Table 2 are based on the fit and do not take into account additional possible errors in the primary mass, such as those introduced by the evolutionary models used to estimate the primary mass.

\section{NACO observations}

A binary separation of about $3 \mathrm{AU}$ or more was predicted for CHXR 74 based on RV data from 2000 to 2006 (Joergens 2008). This corresponds to an angular separation of almost 20 milli arcsec (mas) or more at the distance of Cha I (160$165 \mathrm{pc}$ ). Therefore, it seemed to be within the realms of possibility to directly resolve this very young spectroscopic binary system with current direct imaging facilities. A resolved orbit would yield the orbital inclination and with it an absolute companion mass and absolute semi-major axes as well as an independent measurement of the distance.

For this purpose, we carried out adaptive optics (AO) imaging of CHXR 74 with NAOS Conica (NACO, Lenzen et al. 2003; Rousset et al. 2003) attached to the VLT $8.2 \mathrm{~m}$ YEPUN telescope, on 16 Jan. 2008. Observations were made with IR wavefront sensing (N90C10 dichroic) in $K$-band with the S13 camera providing a pixel scale of $13.3 \mathrm{mas} /$ pixel, and with the target itself as natural guide star (NGS). Data were taken at two instrument rotator angles separated by $90^{\circ}$. At each angle, 11 frames
Table 2. Orbital and physical parameters derived for the best-fit Keplerian model of CHXR 74.

\begin{tabular}{lc}
\hline \hline Parameter & Value \\
\hline$P$ (days) $\ldots \ldots \ldots$ & $4770 \pm 386$ \\
$T($ HJD -2450000$)$ & $1608 \pm 204$ \\
$e \ldots \ldots \ldots \ldots \ldots \ldots$ & 0.0 (fixed) \\
$\omega\left({ }^{\circ}\right) \ldots \ldots \ldots \ldots$ & 0.0 (fixed) \\
$K\left(\mathrm{~km} \mathrm{~s}^{-1}\right) \ldots \ldots \ldots$ & $2.17 \pm 0.14$ \\
$V_{0}\left(\mathrm{~km} \mathrm{~s}^{-1}\right) \ldots \ldots$ & $17.528 \pm 0.17$ \\
\hline$f(m)\left(10^{-3} M_{\odot}\right) \ldots$ & $5.026 \pm 1.061$ \\
$M_{2} \sin i\left(M_{\text {Jup }}\right) \ldots$ & $84 \pm 6$ \\
$M_{2} \sin i\left(M_{\odot}\right) \ldots \ldots$ & $0.08 \pm 0.006$ \\
$a_{1} \sin i(\mathrm{AU}) \ldots \ldots$ & $0.95 \pm 0.08$ \\
$a_{2}(\mathrm{AU}) \ldots \ldots \ldots$ & $2.84 \pm 0.20$ \\
\hline$N_{\text {meas }} \ldots \ldots \ldots \ldots$ & 22 \\
Span $($ days $) \ldots \ldots$ & 4130 \\
$\sigma(\mathrm{O}-\mathrm{C})(\mathrm{m} / \mathrm{s}) \ldots \ldots$ & 395 \\
$\chi_{\text {red }}^{2} \ldots \ldots \ldots \ldots$ & 0.94 \\
\hline
\end{tabular}

Notes. The given parameters are: orbital period, periastron time, eccentricity, longitude of periastron, RV semi-amplitude, system velocity, mass function, lower limit of the companion mass, lower limit of the semi-major axis of the primary, semi-major axis of the companion, number of measurements, time span of the observations, residuals, reduced $\chi^{2}$. A model-dependent primary mass $M_{1}=0.24 M_{\odot}$ was used to derive $M_{2} \sin i$ and $a_{2}$; the given errors of $M_{2} \sin i$ and $a_{2}$ are solely based on the fit. See text for more details.

were taken with jittering applied. Each frame consisted of a single $12 \mathrm{~s}$ exposure, giving a total integration time of $132 \mathrm{~s}$ per angle. The data were reduced and collapsed into a final $0^{\circ}$ and a final $90^{\circ}$ frame using the "jitter" routine of the ESO "eclipse" package.

There is no convincing companion detected to CHXR 74 in the images. The point spread function (PSF) has a FWHM of about 70 mas and is slightly extended in the north/south direction (along close to the $y$-axis in the $0^{\circ}$ image and to the $x$-axis in the $90^{\circ}$ image). The extension direction is clearly distinct from the parallactic angle during the observations $\left(\sim-40^{\circ}\right)$, which excludes the possibility that it is a result of differential atmospheric refraction. Hence, it is in principle possible that this extension is caused by the companion. However, because the signal is rather small, and because some instrumental effects are hard to exclude (e.g., an unfortunate PSF variation between the $0^{\circ}$ and $90^{\circ}$ frames), we do not consider this as a reliable detection. Instead, we used the data to quantify upper limits to the brightness ratio and lower limits to the projected separation by excluding parameters that would cause a stronger impact on the PSF than what we observe, to a statistically significant degree.

This was performed by introducing fake companions in the data at different brightness ratios and separations, and evaluating the signal-to-noise ratio $(S / N)$ of the resulting signature. The effect on the PSF as a function of companion properties is quantified in terms of the PSF width along the extended direction, compared to the width along the perpendicular direction. The procedure is the following: the $0^{\circ}$ and $90^{\circ}$ pair of images of CHXR 74 with no artificial companions are taken as the reference cases of a star with no (adequately significant) companion signature. For this pair of images, we loop through position angles of $0^{\circ}$ through $170^{\circ}$, in steps of $10^{\circ}$. For each angle, a cross-section of the PSF at that angle is sampled through bilinear interpolation along the cut. A Gaussian fit is performed on each cross-section, evaluating in particular the FWHM. The array of FWHMs at $0^{\circ}$ and $90^{\circ}$ are denoted $w_{\text {ref }, 0}$ and $w_{\text {ref }, 90}$, respectively. These are 


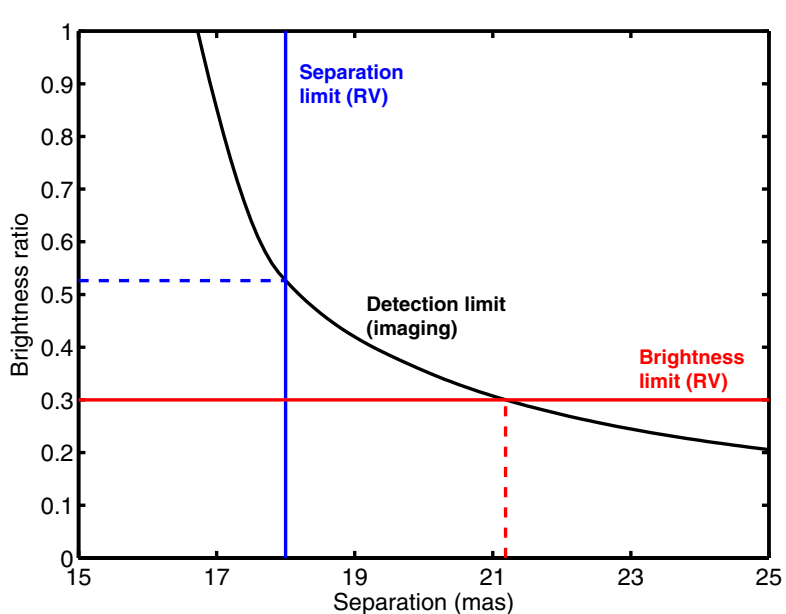

Fig. 3. Detection map of the NACO observations of CHXR 74. Displayed are the parameter space of detectable companions in terms of $K$-band brightness ratio and binary separation. The black curve denotes the detection limit for a $S / N$ of 5 . The blue vertical line represents the minimum binary separation (18 mas) at the time of the NACO observations and the red horizontal line the model-dependent lower limit of the $K$-band brightness ratio, both derived from the RV orbit. See text for more details.

subtracted pairwise to create $\Delta w_{\text {ref }}=w_{\text {ref }, 0}-w_{\text {ref }, 90}$. This means that if the PSF were significantly extended along $50^{\circ}, \Delta w$ would show a sinusoidal-like variation, with a maximum at $50^{\circ}$ and a minimum at $140^{\circ}$. As we discussed above, $\Delta w_{\text {ref }}$ does weakly display such a trend with a peak at close to $0^{\circ}$. The total scatter (i.e., the standard deviation) of $\Delta w_{\text {ref }}$ is taken as the noise level $N$.

We then systematically introduced artificial companions in the images. These are copies of the primary PSF, added at different separations and with different brightness ratios. A grid was used for the sampling that sampled the separation in steps of 0.15 pixels, from 0.15 pixels to 3.00 pixels, and the brightness ratio in steps of 0.05 , from 0.05 to 1.00 . The $\Delta w$ corresponding to a simulated case is denoted $\Delta w_{\text {sim }}$. For each case we evaluated the signal $S$ as the rms of the $\Delta w_{\text {sim }}-\Delta w_{\text {ref }}$ components. We set $S / N>5$ as the criterion for detectability. In Fig. 3 we show the parameter ranges that remain allowed and can be excluded, respectively, on the basis of the images.

\section{Conclusions}

We confirm that CHXR 74 (M4.25) is a very low-mass pre-main sequence spectroscopic binary based on new RV measurements obtained between 2008 and 2011 with UVES at the VLT. The Kepler orbit that fits the combined RV data set (2000-2011) best has an orbital period of 13.1 years and zero eccentricity. A companion minimum mass $M_{2} \sin i$ of $0.08 M_{\odot}$ was derived by using a model-dependent mass estimate for the primary of $0.24 M_{\odot}$. The binary separation $\left(a_{1} \sin i+a_{2}\right)$ for an inclination of $90^{\circ}$ is $3.8 \mathrm{AU}$, which corresponds to 23 mas at the distance of Cha I (160-165 pc). Complementing the RV measurements, high-resolution AO images of CHXR 74 were taken with NACO at the VLT with the aim of directly resolving the binary. While there are marginal signs of an extended PSF, we have no convincing companion detected to CHXR 74 in these images.

In the following, we investigate the parameter space of binary properties that is allowed for CHXR 74 given the presented UVES and NACO observations. First, we calculate the absolute companion mass $M_{2}$ for different inclinations based on the RV orbit and taking the companion's luminosity into account. For this calculation, $M_{2}$ was derived directly from the mass function $f(m)$ rather than using the value of $M_{2} \sin i$ given in Table 2, which is for non-negligible companion masses valid only for large inclination angles. The luminosity ratio $L_{2} / L_{1}$ and the primary mass $M_{1}$ were then estimated for different inclinations by employing low-mass evolutionary model tracks (Baraffe et al. 1998) and assuming the coevality of the binary components. For the luminosity of the unresolved system and its effective temperature, the values determined by Luhman (2007, cf. Sect. 2) were used. While they correspond to a mass estimate of $0.25 M_{\odot}$, the fact that the secondary of CHXR 74 contributes significantly to the total luminosity leads to a lower primary mass estimate depending on the inclination.

At minimum, i.e. for an inclination $i=90^{\circ}$, the mass of the companion is estimated to be $0.08 M_{\odot}$. In this case the luminosity ratio $L_{2} / L_{1}$ is 0.30 and the primary has a mass of about $0.24 M_{\odot}$. For smaller inclination angles, the mass and luminosity of the companion increases, e.g., for $i=60^{\circ}$, a companion mass of $0.10 M_{\odot}$ is derived. In this case, $L_{2} / L_{1}$ is estimated to 0.36 and $M_{1}$ to about $0.24 M_{\odot}$. The limiting case is to consider a companion that is as luminous as the primary (i.e., $L_{1}=L_{2}=L_{\mathrm{tot}} / 2$ ), although we show in the next paragraph that this is unrealistic. Comparison with evolutionary tracks shows that then both components would have a mass of $M_{1}=M_{2} \approx 0.23 M_{\odot}$. This implies a minimum orbital inclination of $27^{\circ}$, as derived from the mass function.

We show in this paragraph that the range of possible inclinations and companion masses constrained by the RV data can be further restricted based on the NACO observations. The binary separation of CHXR 74 on the date of our NACO observations is predicted to be $3.0 \mathrm{AU}$ at minimum $\left(i=90^{\circ}\right)$ based on the RV orbit solution (Reffert, pers. comm.). This corresponds to an angular separation of 18 mas at the distance of Cha I. Considering the non-detection in the NACO observations and the derived detectable $K$-band brightness ratio as function of the actual separation (black curve in Fig. 3), the minimum separation of 18 mas (blue vertical line in Fig. 3) can be translated into an upper limit for the $K$-band brightness ratio of the two components in CHXR 74 of 0.53 . This brightness ratio is applied to divide the $K_{\mathrm{S}}$-band magnitude measured for the unresolved system $\left(m_{K}=10.21 \mathrm{mag}\right)$ among the two components $\left(m_{K, 1} \leq 10.67 \mathrm{mag}, m_{K, 2} \geq 11.36 \mathrm{mag}\right)$. Comparing again with evolutionary tracks yields an upper limit for the companion mass of $0.14 M_{\odot}$ and a lower limit for the primary mass of $0.23 M_{\odot}$. This requires the orbital inclination to be $\geq 40^{\circ}$ (again derived directly from the mass function rather than using $M_{2} \sin i$ ). Therefore we conclude from the combined UVES and NACO observations that the secondary of CHXR 74 has a mass within the range of about 0.08 and $0.14 M_{\odot}$.

We note that the combined RV and imaging data allow us also to derive an upper limit for the binary separation at the time of the NACO observation of slightly above 21 mas (3.4 AU). This follows from the minimum mass $M_{2} \sin i$ from the RV orbit $\left(0.08 M_{\odot}\right)$, which can be converted into a model-dependent lower limit for the $K$-band brightness ratio of about 0.3 , which in turn can be translated into a separation via the NACO detection map (red horizontal line in Fig. 3). This means, on one hand, that we have apparently just missed the companion in the NACO observations. On the other hand, it demonstrates that the orbital period cannot be dramatically longer than the value determined from the RV data (13.1 yr), which sample about $87 \%$ of the orbit. 
The astrometric space mission Gaia might be able to monitor the astrometric orbit of the primary in CHXR 74. While the minimum semi-major axis of the primary is $0.95 \mathrm{AU}$ ( 6 mas), the astrometric signature is smaller for a non-negligible luminosity of the companion because the astrometric orbit corresponds to the orbit of the photocenter around the center of mass. Taking this into account, we predict an astrometric signature of the primary of CHXR 74 between 0.4 and 0.2 mas for the inclination range between $90^{\circ}$ and $40^{\circ}$. If the astrometric orbit can be determined, it will yield in combination with the presented RV orbit solution, the absolute mass of the companion and an independent distance measurement. This would provide a valuable dynamical mass measurement at young ages in the poorly constrained mass regime of $<0.3 M_{\odot}$.

Acknowledgements. We acknowledge the excellent work of the ESO staff at Paranal, who took the data presented here in service mode. We thank S. Reffert for calculating the separation prediction at the time of the NACO observations. Furthermore, we are grateful for comments from the referee M. Simon and the editor T. Forveille that helped to improve the paper. Part of this work was funded by the ESF in Baden-Württemberg.

\section{References}

Baraffe, I., Chabrier, G., Allard, F., \& Hauschildt, P. H. 1998, A\&A, 337, 403 Basri, G., \& Martín, E. L. 1999, ApJ, 118, 2460

Blake, C. H., Charbonneau, D., White, R. J., et al. 2008, ApJ, 678, L125

Comerón, F., Rieke, G. H., \& Neuhäuser, R. 1999, A\&A, 343, 477

Dekker, H., D’Odorico, S., Kaufer, A., Delabre, B., \& Kotzlowski, H. 2000, in SPIE, ed. M. Iye, \& A. Moorwood, 534, 4008

Joergens, V. 2006, A\&A, 446, 1165

Joergens, V. 2008, A\&A, 492, 545

Joergens, V., \& Müller, A. 2007, ApJ, 666, L113

Joergens, V., Müller, A., \& Reffert, S. 2010, A\&A, 521, A24

Konopacky, Q. M., Ghez, A. M., Barman, T. S., et al. 2010, ApJ, 711, 1087

Lenzen, R., Hartung, M., Brandner, W. et al. 2003, SPIE, 4841, 944

Luhman, K. L. 2004, ApJ, 614, 398

Luhman, K. L. 2007, ApJS, 173, 104

Luhman, K. L., Allen, L. E., Allen, P. R. et al. 2008, ApJ, 675, 1375

Mathieu, R. D., Baraffe, I., Simon, M., Stassun, K. G., \& White, R. 2007, in Protostars and Planets V, ed. by B. Reipurth, D. Jewitt, \& K. Keil (Tucson: University of Arizona Press), 411

Rousset, G., Lacombe, F., Puget, P., et al. 2003, SPIE, 4839, 140

Simon, M., Bender, C., \& Prato, L. 2006, ApJ, 644, 1183

Stassun, K. G., Mathieu, R. D., \& Valenti, J. A. 2006, Nature, 440, 311

Zapatero Osorio, M. R., Lane, B. F., Pavlenko, Ya., et al. 2004, ApJ, 615, 958

Zechmeister, M., \& Kürster, M. 2009, A\&A, 496, 577 